\title{
Molecular detection of hepatitis E virus (HEV) in liver biopsies after liver transplantation
}

Ulrike Protzer ${ }^{1,2}$, Friederike Böhm³ ${ }^{3}$ Thomas Longerich ${ }^{4}$, Judith Seebach ${ }^{1}$, Mojdeh Heidary Navid ${ }^{5}$, Juliane Friemel ${ }^{3}$, Ewerton Marques-Maggio ${ }^{3}$, Marion Bawohl ${ }^{3}$, Mathias Heikenwalder ${ }^{1,2}$, Peter Schirmacher ${ }^{2,4}$, Philipp Dutkowski ${ }^{6}$, Pierre-Alain Clavien ${ }^{6}$, Peter Schemmer ${ }^{7}$, Paul Schnitzler ${ }^{5}$, Daniel Gotthardt ${ }^{8}$, Beat Müllhaupt ${ }^{9}$ and Achim Weber ${ }^{3}$

${ }^{1}$ Institute of Virology, Technische Universität München/Helmholtz Zentrum München, Munich, Germany;

${ }^{2}$ German Center for Infection Research (DZIF), Munich and Heidelberg Sites, Braunschweig, Germany;

${ }^{3}$ Institute of Surgical Pathology, University Zurich, Zurich, Switzerland; ${ }^{4}$ Institute of Pathology, University

Hospital Heidelberg, Heidelberg, Germany; ${ }^{5}$ Department of Infectious Diseases, Virology, University Hospital Heidelberg, Heidelberg, Germany; ${ }^{6}$ Clinics of Visceral and Transplant Surgery, University of Zurich, Zurich, Switzerland; ' Department of General and Transplant Surgery, University Hospital Heidelberg, Heidelberg, Germany; ${ }^{8}$ Department of Gastroenterology, University Hospital Heidelberg, Heidelberg, Germany and ${ }^{9}$ Clinics of Hepatology and Gastroenterology, University of Zurich, Zurich, Switzerland

\begin{abstract}
We aimed to determine the rate of hepatitis E virus (HEV) infection, a recently increasingly recognized disease in the Western world, in liver transplant patients by direct molecular testing of liver tissue. A RT-PCR assay was designed for detecting the HEV open reading frame (ORF) 2/3 gene region in formalin-fixed, paraffin-embedded tissues, and applied to all liver biopsies $(n=683)$ taken 4 weeks or later from all patients $(n=282)$ after liver transplantation of two large academic centers. HEV-RNA was detected in ten biopsies from four different patients (rate: 1\%). Histology in early HEV infection was variable including cases with only few hepatocellular apoptoses, no or only minute inflammation. Hepatitis lasted for at least 6 months in $3 / 4$ patients. Serologic testing for HEV-RNA in a subcohort (159 patients) was positive in five patients (rate: $3 \%$ ), resulting in an overall HEV detection rate of $3 \%(8 / 282)$. In case both liver tissue and sera of a patient were available from the same time period, all cases tested positive in one material were also tested positive in the other material, respectively. All patients had de novo autochthonous infection with HEV genotype 3. Our data confirm that HEV infection is a relevant cause of liver injury after liver transplantation. Molecular testing for HEV in routinely processed transplant liver biopsies is powerful for evaluating patients with elevated transaminases of unknown origin. Histology of HEV infection under immunosuppression in the early phase is distinct from HEV infection in immunocompetent individuals.
\end{abstract}

Modern Pathology (2015) 28, 523-532; doi:10.1038/modpathol.2014.147; published online 21 November 2014

Hepatitis E virus (HEV), a non-enveloped singlestranded hepatotropic RNA virus, is the infectious agent causing hepatitis E. Humans can be infected by HEV genotypes $1,2,3$, and 4, which show a worldwide variable distribution and prevalence. Genotype 1, most prevalent in Asia, and genotype 2, most prevalent in Africa and Central America, are restricted to humans. Genotype 3, prevalent in industrialized countries of North America and

Correspondence: Professor A Weber, MD, Institute for Surgical Pathology, University Hospital Zurich, Schmelzbergstrasse 12, CH-8091 Zürich, Switzerland.

E-mail: achim.weber@usz.ch

Received 28 June 2014; revised 25 August 2014; accepted 2 September 2014; published online 21 November 2014
Europe, as well as genotype 4, most prevalent in Eastern Asia, in contrast are zoonotic. $^{1-5} \mathrm{HEV}$ genotype 3 has been detected, eg, in pigs, wild boars, deer, and rodents (with swine considered as the main reservoir), and can be transmitted via porcine meat. ${ }^{6}$ Among humans, HEV undergoes fecal-oral transmission, mostly by contaminated drinking water but also by smear infection. For a long time, hepatitis $\mathrm{E}$ has been regarded a disease restricted to warmer, resource-low countries with poor sanitation, but it is now increasingly recognized also in industrialized countries. ${ }^{3,7} \mathrm{HEV}$ infections are far more frequent than previously thought ${ }^{8}$ causing $>25 \%$ of sporadic hepatitis in some regions and in the past were frequently interpreted as druginduced liver damage. ${ }^{9,10} \mathrm{HEV}$ infection normally is 
either asymptomatic or causes self-limited, acute, icteric hepatitis taking a mild course. However, severe disease with fatal outcome is observed in pregnant women ${ }^{11}$ or in patients with preexisting chronic liver diseases. ${ }^{12,13}$ In immunosuppressed patients especially after (solid) organ transplantation, HEV infection may become chronic. ${ }^{14-16}$

Unexplained liver injury after liver transplantation prompts a diagnostic work-up which in many instances includes a liver biopsy ${ }^{17}$ and also testing for (viral) infections, but screening for HEV is still not routinely done in Europe. Serological testing for HEV infection can be performed by screening for anti-HEV antibodies. However, this may be hampered by a significant variation in sensitivity and specificity of anti-HEV antibody-based assays ${ }^{18}$ and the fact that seroconversion often is delayed in immunosuppressed patients. Interpretation of positive test results is difficult due to high antiHEV antibody prevalence (up to $20 \%$ ) even in developed countries. Direct proof of HEV RNA is usually performed by PCR and essential to prove acute infection. However, HEV-RNA assays in blood or stool samples show variable sensitivity and lack standardization. ${ }^{19}$ Furthermore, viremia and even more excretion of HEV in stool is only observed for a limited time span indicating that detection in liver tissue, ie, at the site of viral replication, may be more reliable.

We therefore aimed at testing for HEV in liver tissue samples including formalin-fixed, paraffinembedded tissue and at comparing its sensitivity to anti-HEV and HEV RNA testing in patient serum. We retrospectively tested for HEV RNA in all patients who had been submitted to liver biopsy at least 4 weeks after liver transplantation between 2004/2005 and 2010 in two large academic centers, and found a substantial number of patients who had acquired HEV infection.

\section{Materials and methods}

\section{Patients and Liver Tissue Samples}

Included were all patients (Table 1) who had undergone liver transplantation between 2005 and 2010 in the Swiss Hepatopancreato-Biliary (Swiss
HPB) Center, University of Zurich, Switzerland, and between October 2004 and 2010 in a German center, ie, University Hospital Heidelberg.

In both centers, liver biopsies were performed as reflex (and not per protocol) testing. All liver biopsies taken 4 weeks or later after liver transplantation and performed until April 2012 were analyzed. If patients had been tested positive for HEV, then clinical parameters were retrospectively retrieved from the patients' files. The study was performed in line with the ethical guidelines of the 1975 Declaration of Helsinki, and in line with local ethics regulations (Retrospective Data Analysis; Clinical Trials Center, University Hospital Zurich). Local ethics committee approval was obtained for the Zurich patients' cohort (KEK-ZH-Nr. 2013-0504) as well as the Heidelberg patients' cohort (approval no. 206/2005).

\section{Histopathologic Evaluation of Liver Tissues}

For patients tested positive for HEV, histological slides of the liver biopsies taken for routine diagnostic work-up were re-evaluated. These included hematoxylin and eosin stains, sirius red (University Hospital Zurich) or modified Gomori's stain (University Hospital Heidelberg) for determination of fibrosis, periodic acid-Schiff (PAS) after diastase digestion, Perl's stain, and in some cases also immunostains for viral infections (human cytomegalovirus and adenovirus). HEV-positive cases were independently evaluated by two experienced hepatopathologists (AW and TL). The following parameters were evaluated: spotty necrosis (none: - ; few/some: + ; many: ++ ), apoptosis (none: - ; few/some: + ; many: ++ ), confluent necrosis (none: - ; focal: + ; bridging: ++ ), portal inflammation (none: - ; mild: + ; moderate: ++ ; severe: +++ ), interface activity (none: - ; focal: + ; diffuse: ++ ), bilirubinostasis (none: - ; mild: + ; prominent: ++ ), cholatestasis (none: - ; mild: + ; prominent: ++ ), ductular reaction (none: - ; mild: + ; prominent: ++ ), degenerative changes in interlobular bile ducts (none: -; focal/mild: +; moderate: ++ ; diffuse/severe: +++ ), fibrosis ( $\mathrm{p}$ : portal; s: septal; b: bridging, a: architectural distortion, c: cirrhosis), and fatty change (none: - ; mild

Table 1 Patient's characteristics

\begin{tabular}{|c|c|c|c|}
\hline Center & Swiss (Zurich) & German (Heidelberg) & Zurich + Heidelberg \\
\hline Inclusion period (year of liver transplantation) & $2005-2010$ & $2004-2010$ & $2004-2010$ \\
\hline Number of liver transplantations/patients with liver transplantation & $197 / 186$ & $647 / 549$ & $844 / 735$ \\
\hline Liver transplantation (patients) with liver biopsy ${ }^{\mathrm{a}}$ & $125(123)$ & $173(159)$ & $298(282)$ \\
\hline Total number of liver biopsies tested & 354 & 329 & 683 \\
\hline Patients positive for hepatitis E virus (HEV) & 3 & 1 & 4 \\
\hline Total number of positive liver biopsies & $9(5 / 2 / 2)$ & 1 & $10(5 / 2 / 2 / 1)$ \\
\hline
\end{tabular}

${ }^{\mathrm{a}}$ Liver biopsy performed 4 weeks or later after liver transplantation. 
$(<25 \%):+$; moderate (25-49\%): + + ; fatty liver $(>49 \%):+++$. Acute rejection was evaluated according to the Banff classification system. ${ }^{20}$ For HEV-positive cases, previous liver specimens including the liver explant and biopsies taken before 4 weeks post-LT were histologically evaluated and subjected to reverse transcription PCR (RT-PCR) testing.

\section{HEV RNA Detection by Semi-Nested RT-PCR and Sequence Analysis}

RNA was extracted from three $10 \mu \mathrm{M}$ sections of formalin-fixed, paraffin-embedded liver biopsies after de-paraffinizing using either Trizol ${ }^{\circledR}$ reagent, or the Qiagen tissue extraction kit according to the manufacturer's instructions and dissolved in $22 \mu \mathrm{l}$ $\mathrm{H}_{2} \mathrm{O}$. For detection of HEV, we designed primer pairs hybridizing to HEV genomic sequences highly conserved between genotype 1 to 4 sequences reported in Genebank. A semi-nested RT-PCR approach was chosen to target the open reading frame (ORF) 2/3 region of the HEV genome (Figure 1a) and was validated according to International Organisation for Standardisation (ISO) standard ISO15189. Oligonucleotide primers were HEV-4-S1: 5'-CGG(G/ T)TGGAATGAATAACATGT-3', HEV-4 A1: 5'-CGCC AAGCGGAGCCG AG-3', HEV-4-A2: 5'-GGTTGGTT GGATGAATATAGG- $3^{\prime}$. In all, $5 \mu$ l RNA was reverse transcribed into $10 \mu \mathrm{l}$ cDNA using Superscript III Reverse Transcriptase (Invitrogen). In all, $5 \mu \mathrm{l}$ cDNA was subjected to first-round PCR yielding a product of 312 base pairs (bp). Three of $50 \mu$ l of the first PCR were subjected to nested PCR amplification leading to a product of $228 \mathrm{bp}$ (Figure $1 \mathrm{~b}$ ). Sensitivity of the nested PCR was determined to be 4-5 cDNA copies using an external plasmid standard. The dynamic PCR range was between 2x10e1 and 2x10e9 copies of HEV cDNA per PCR, and a secure detection threshold of 22 copies of HEV cDNA per PCR was defined. Specificity was determined by testing 15 negative samples of different origins (sera and cells/ tissues), and samples positive for hepatitis A and D viruses, parvo-, noro-, polio-, and adenoviruses. RT-PCR products were submitted to direct Sanger sequencing to confirm specificity and to define the HEV genotype. Sequences were aligned to publicly available HEV reference sequences using the BLAST program (http://www.ncbi.nlm.nih.gov). BioEdit software was used for sequence comparison.

\section{Serologic Testing for HEV}

Results of serologic testing for HEV already performed as part of clinical work-up of patients were retrospectively retrieved from patients' files. Whereas sera of patients of the Swiss cohort were not available for additional testing, sera from patients of the German cohort were retrospectively tested for HEV antibodies, and if positive also for
HEV RNA. Anti-HEV IgG and IgM were tested using an ELISA (recomWell HEV IgG, recomWell HEV IgM, Mikrogen, Neuried, Germany) and confirmed by immunoblot analysis (recomLine HEV IgG/IgM, Mikrogen, Neuried, Germany). Both assays were performed according to the manufacturer's instructions. HEV RNA was tested by RT-PCR after RNA extraction from sera using the same amplification protocol as applied for formalin-fixed, paraffinembedded material.

\section{Results}

Liver Transplantation and Liver Biopsy, HEV Testing, Sequencing and Genotyping

Between 2005 and 2010, 197 liver transplantations were performed on 186 patients at the Swiss HPB Center, University of Zurich. Between July 2004 and May 2011, 647 liver transplantations were performed on 549 patients at the University Hospital Heidelberg. In most cases elevated transaminases set the indication for liver biopsy in 125 of the 197 liver transplantations at the Swiss HPB Center (on a total of 123 patients), and in 173 of the 647 liver transplantations at the University of Heidelberg (on a total of 159 patients) after liver transplantation. Thus, 683 (Zurich: 354, Heidelberg: 329) liver biopsies were tested for HEV RNA from a total of 298 liver transplantations (on a total of 282 patients). Indications for liver transplantation are summarized in Table 2.

HEV RNA was detected in ten biopsies from four different patients (patient 1 (Zurich): $n=5$, Figure $1 b$; patient 2 (Zurich): $n=2$; patient 3 (Zurich): $n=2$; patient 4 (Heidelberg): $n=1$; see Table 3 ), of which only two (patient 1 and patient 4) had been detected by serology screening before. This corresponds to a rate of $\mathrm{HEV}$ infections detected in liver biopsies of $1 \%$ in relation to the number of patients with liver transplantations $(4 / 282)$, and $2 \%$ in relation to the number of liver biopsies (10/683), respectively. Biopsies were taken between 1-82 months after liver transplantation in Zurich (mean 16 months) and 1-64 months after liver transplantation in Heidelberg (mean 13 months). In all four patients tested positive, no HEV RNA was detected in the liver explant, and all had at least one negative biopsy in the early post-transplant period. In addition, for patient 1 and patient 2, donor biopsies were available that also tested negative for HEV. This indicates that all four patients acquired HEV infection de novo after LT. In patient 2 and patient 3 , neither HEV antibodies nor HEV RNA was detectable in serum when diagnosis was established by PCR testing of liver biopsies.

Direct sequencing of RT-PCR products confirmed the specificity of amplification, and revealed nucleotide sequences specific for the ORF $2 / 3$ gene region of HEV genotype 3 for all amplicons. 
a

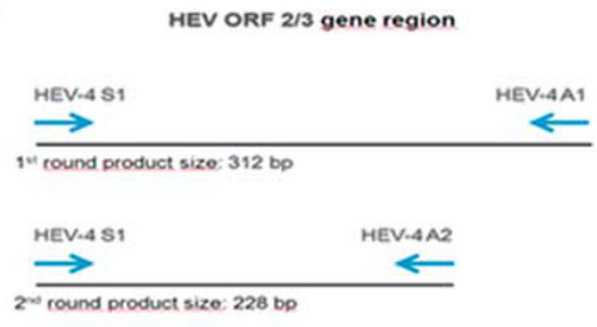

b

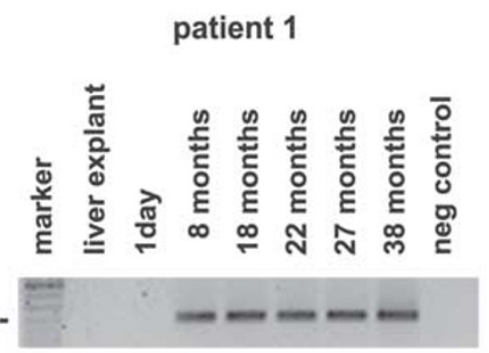

C

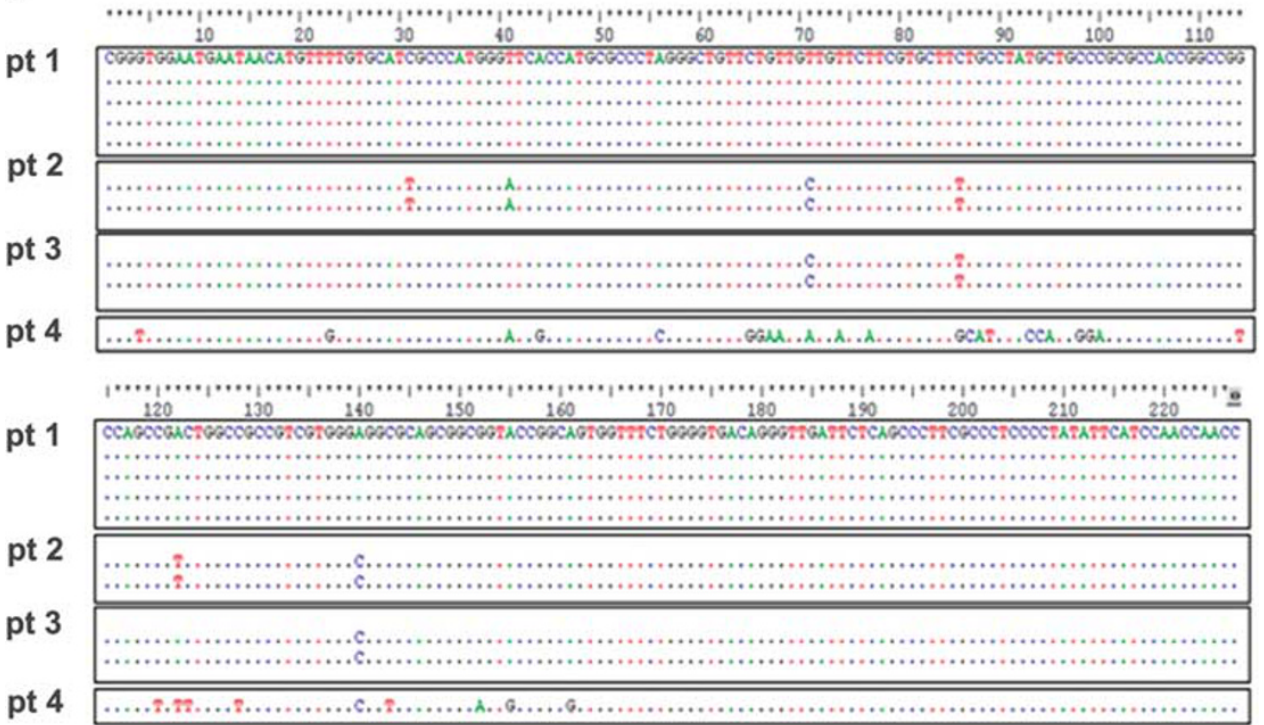

Figure 1 Reverse transcription (RT)-PCR testing for hepatitis E virus (HEV). (a) Scheme of semi-nested RT-PCR targeting the HEV open reading frame (ORF) 2/3 gene region. (b) Liver tissues available from patient 1: No amplification of HEV-specific sequences in the liver explant and day 1 biopsy, but in all following liver biopsies indicating chronic HEV infection acquired post-LT. (c) Direct sequencing of PCR products revealed HEV genotype 3 in all amplicons. Nucleotide sequences of the ORF $2 / 3$ gene region varied among the four different patients, but were invariable comparing amplicons from different samples of the same patients.

Sequence alignment of the 10 amplicons (Figure 1c) showed inter-individual sequence variability among the 4 patients of at least 3 of the 228 nucleotides. In contrast, nucleotide sequence in this gene region did not vary intra-individually which in case of patient 1 comprised HEV amplicons from biopsies taken at intervals of up to 30 months.

\section{Correlation with Serologic Testing}

In total, 159 sera from patients of the German cohort were tested at their most recent visit for HEV IgM and IgG antibodies. Anti-HEV antibodies were found in 28 of the 159 patients of the Heidelberg cohort (18\%). From 27 of anti-HEV positive patients, sera before liver transplantation were available for retrospective analysis. In all, 21/27 tested positive for anti-HEV but negative for HEV RNA indicating that they resolved HEV infection before liver transplantation. Five patients experienced anti-HEV seroconversion around or after liver transplantation. In one patient testing positive for anti-HEV after liver transplantation no serum before liver transplantation was available, so that the time point of seroconversion could not be determined. However, HEV RNA testing in serum samples taken after liver transplantation was negative. We therefore rated this also as HEV infection before liver transplantation. Thus, 5/159 patients (3\%) of the German cohort had acquired HEV infection around or after liver transplantation.

\section{Liver Histology}

Clinical features and course of the four patients tested positive for HEV in liver biopsies after liver transplantation are shown in Figures 2-4. The spectrum of liver diseases leading to transplantation comprised cirrhosis due to $\alpha 1$-anti-trypsin deficiency, chronic hepatitis B virus (HBV) infection, and alcohol abuse as well as a case of drug-induced fulminant liver failure. HEV-positive biopsies revealed a broad spectrum of liver pathologies. Morphologic patterns varied not only among the 
different patients, but also among biopsies taken from the same patient during the course of infection (Figures 2-4; Table 3). Patient 1 had the highest number of HEV-positive liver biopsies $(n=5)$ and the longest follow-up period (30 months). The first liver biopsy taken during HEV infection (8 months post liver transplantation) revealed only few hepatocellular apoptoses, but no signs of rejection, inflammation, bile duct changes, or fibrosis

Table 2 Indications for liver transplantation

\begin{tabular}{|c|c|}
\hline Indication for liver transplantation & $\begin{array}{l}\text { Number of } \\
\text { patients }\end{array}$ \\
\hline Chronic hepatitis C virus (HCV) infection & 54 \\
\hline $\begin{array}{l}\text { Chronic HCV infection and hepatocellular } \\
\text { carcinoma (HCC) }\end{array}$ & 19 \\
\hline Chronic HCV infection and alcohol & 4 \\
\hline Chronic hepatitis B virus (HBV) infection & 16 \\
\hline Fulminant HBV infection & 1 \\
\hline Chronic HBV infection and HCC & 2 \\
\hline Alcohol & 28 \\
\hline Alcohol and HCC & 4 \\
\hline $\begin{array}{l}\text { Acute/subacute liver failure } \\
\text { (not otherwise specified) }\end{array}$ & 15 \\
\hline HCC & 32 \\
\hline Cholangiocellular carcinoma (CCC) & 3 \\
\hline Transplant failure & 4 \\
\hline Wilson disease & 8 \\
\hline$\alpha 1$-Antitrypsin deficiency & 1 \\
\hline Hemochromatosis & 2 \\
\hline Primary sclerosing cholangitis (PSC) & 11 \\
\hline Primary biliary cirrhosis (PBC) & 2 \\
\hline PBC and HCC & 2 \\
\hline Autoimmune hepatitis & 5 \\
\hline Cystic fibrosis & 1 \\
\hline Amyloidosis & 1 \\
\hline Cryptogenic cirrhosis & 12 \\
\hline Others & 55 \\
\hline Sum & 282 \\
\hline
\end{tabular}

(Figure 2, left). Biopsies taken between 18 and 38 months post liver transplantation showed progressively established chronic hepatitis, and development of portal and septal fibrosis (Figure 2, middle to right). Patient 2 had two HEV-positive liver biopsies at an interval of 3 months (Figure 3a). The first biopsy (4 months post liver transplantation) revealed few hepatocellular apoptoses, spotty necroses, no significant inflammation, unremarkable interlobular bile ducts, and at most mild portal fibrosis. The second biopsy (7 months post liver transplantation) showed some hepatocellular apoptoses and many spotty necrosis, mild portal and focal interface inflammation, moderate bile duct damage, mild ductular rejection, and minimal portal fibrosis. In addition, there were signs of mild acute cellular reaction. Patient 3 had two HEV-positive liver biopsies taken at an interval of 2 weeks (Figure $3 \mathrm{~b}$ ). The first biopsy (2 months post liver transplantation) revealed some hepatocellular apoptoses and spotty necroses, moderate portal and focal interface inflammation, moderate bile duct damage, focal ductular reaction, and no fibrosis. There were also many ceroid-laden macrophages, mild focal endothelialitis, and signs of mild acute cellular rejection. The second biopsy (2 1/2 months post liver transplantation, following rejection therapy) showed only few spotty necroses, mild inflammation in some portal tracts, no significant bile duct damage or ductular reaction, and no fibrosis. Endothelialitis and signs of acute cellular rejection were no longer evident but regenerative changes. Only one HEV-positive biopsy (20 months post liver transplantation) was available for patient 4 revealing some hepatocellular apoptoses and many single-cell necrosis, inflammation in some portal tracts and focal interface activity, moderate degenerative bile

Table 3 Clinical parameters of patients with liver biopsies tested positive for hepatitis E virus (HEV) RNA

\begin{tabular}{|c|c|c|c|c|}
\hline Patient no. & Patient 1 & Patient 2 & Patient 3 & Patient 4 \\
\hline $\begin{array}{l}\text { Age (time of liver } \\
\text { transplantation) }\end{array}$ & 55 years & 29 years & 35 years & 48 years \\
\hline Sex & Male & Female & Male & Female \\
\hline Center & Swiss (Zurich) & Swiss (Zurich) & Swiss (Zurich) & German (Heidelberg) \\
\hline $\begin{array}{l}\text { Indication for liver } \\
\text { transplantation }\end{array}$ & $\begin{array}{c}\alpha 1-\text { Antitrypsin } \\
\text { deficiency, } \\
\text { cirrhosis, hepatocellular } \\
\text { carcinoma (HCC) }\end{array}$ & $\begin{array}{l}\text { Drug-induced fulminant liver } \\
\text { failure }\end{array}$ & $\begin{array}{l}\text { Cirrhosis, chronic } \\
\text { hepatitis B virus } \\
\text { (HBV) infection }\end{array}$ & $\begin{array}{c}\text { Cirrhosis, alcohol } \\
\text { abuse }\end{array}$ \\
\hline $\begin{array}{l}\text { Months since liver } \\
\text { transplantation }\end{array}$ & 8 & 3 & 2 & 19 \\
\hline Immunosuppressive therapy & $\begin{array}{c}\text { Initially cyclosporin A, } \\
\text { later tacrolimus }\end{array}$ & Tacrolimus & $\begin{array}{l}\text { Tacrolimus, } \\
\text { sirolimus }\end{array}$ & $\begin{array}{l}\text { Tacrolimus, } \\
\text { mycophenolate } \\
\text { mofetil }\end{array}$ \\
\hline $\begin{array}{l}\text { Maximal alanine } \\
\text { aminotransferase (ALT; U/l) }\end{array}$ & 332 & 141 & 1743 & 139 \\
\hline $\begin{array}{l}\text { Maximal asparate } \\
\text { aminotransferase (AST; U/l) }\end{array}$ & 310 & 100 & 515 & 78 \\
\hline Chronicity & Yes & No & No & No \\
\hline Course of infection & Persistent & Resolving & Resolving & Resolving \\
\hline
\end{tabular}

${ }^{\mathrm{a}}$ Monitored during time of documented infection. 

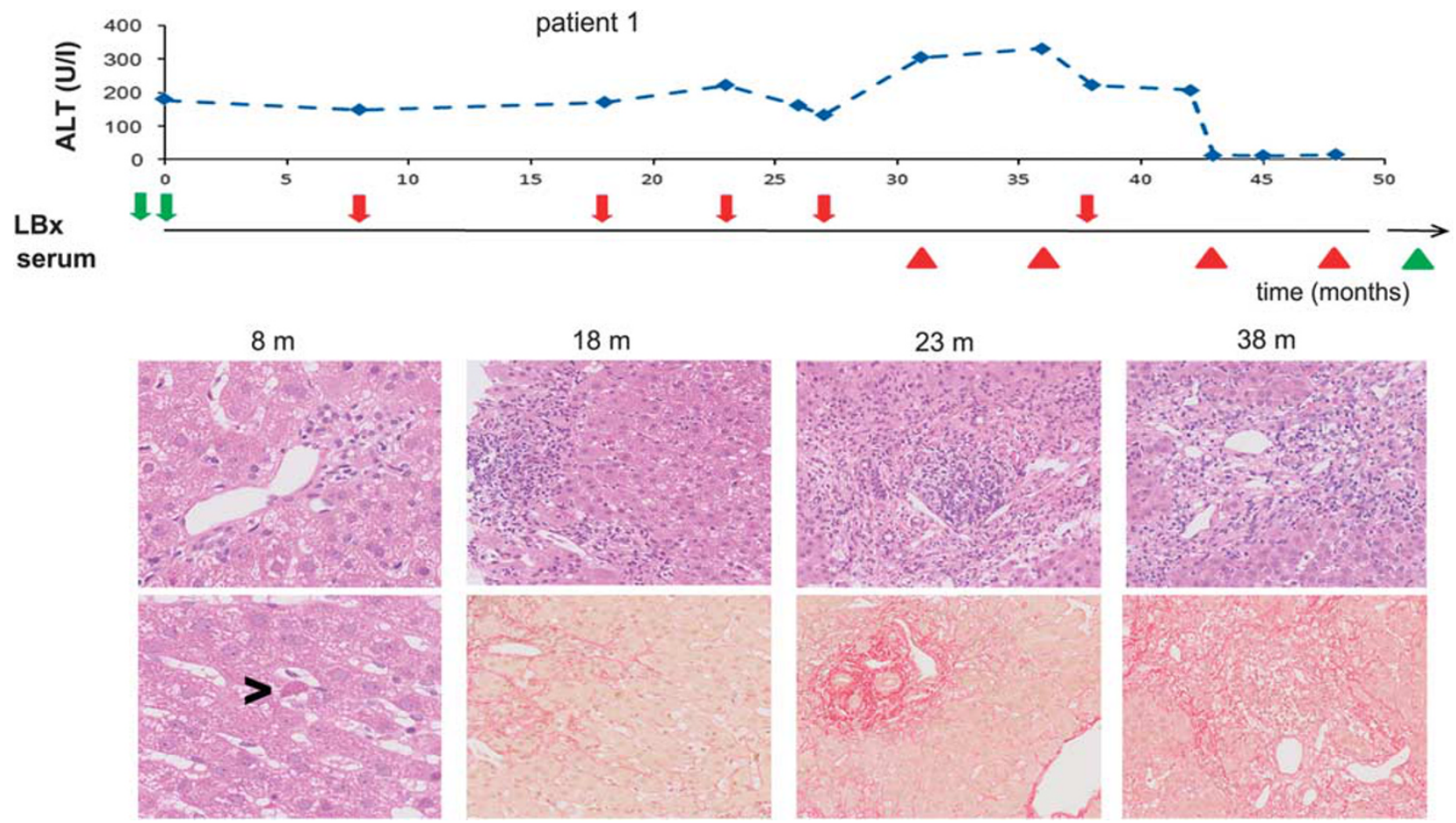

Figure 2 Clinical course and histopathological patterns of hepatitis E virus (HEV) infection after liver transplantation for patient 1. Elevated alanine aminotransferase (ALT) and aspartate aminotransferase (AST) levels persisted for $>40$ months. De novo HEV infection was first detectable in a liver biopsy of 8 months post liver transplantation. Transaminase levels later normalized, but follow-up HEV testing in sera remained positive. Liver histology 8 months post liver transplantation revealed only spotty hepatocyte necroses, but chronic hepatitis and fibrosis developed in the course of the disease. Arrow: liver biopsy, arrow head: serum testing; red: positive for HEV, green: negative for HEV.

duct changes, mild ductular reaction, and portal fibrosis with focal porto-portal bridging. In addition, portal lipoid-storing macrophages were found as well as Kupffer cell siderosis (Figure 4). Of note, cholestasis, bilirubinostasis, or lobular disarray was not found in any of the 10 liver biopsies tested positive for HEV.

\section{Clinical Course}

Of the five patients of the German cohort who newly acquired HEV infection, one acquired the infection shortly before liver transplantation but cleared HEV. In two patients of the German cohort and all four patients of the Swiss cohort, HEV was acquired and eventually cleared after liver transplantation. The two patients from Heidelberg with retrospectively diagnosed self-limiting HEV infection had no significant worsening of liver function. Therefore, no follow-up liver biopsy was taken. Two patients (patient 1 and patient 4, Figures 2 and 4) developed a persistent HEV infection for at least 6 months. On the basis of information yielded from clinical monitoring, transaminase levels, molecular testing for HEV RNA in liver biopsies and in blood, it became evident that patient 1 still carried HEV 42 months post liver transplantation before he finally cleared the virus. Patient 4 had developed chronic HEV infection and was tested repeatedly positive for HEV RNA over 18 months until the last follow-up. One patient showed the clinical picture of acute hepatitis when testing positive for HEV RNA in serum and seroconverting to anti-HEV. He had already cleared HEV RNA from blood and liver after 3 months, when he developed severe chronic cholestasis and progressive ischemic cholangiopathy. Two months later he was diagnosed with ischemic necrosis and incomplete liver cirrhosis, liver function deteriorated and he required re-transplantation. It remained unclear whether at all, and if so to which extent HEV infection had contributed to this clinical course.

All patients had elevated transaminase levels during the time of HEV infection. Patient 1 and patient 4 showed intermittently normal transaminase levels despite persistent HEV infection (Figures 2 and 4). Since patient 1 developed progressive fibrosis, and HEV RNA was still detectable even after reduction of immunosuppression, the patient was treated with Ribavirin (1200 mg daily for 12 weeks). ${ }^{21,22}$ Four weeks after starting treatment transaminase levels normalized. However, HEV RNA was still detectable 3 months after therapy, but had finally been cleared 1 year later. Thus, all patients finally cleared HEV infection. 
a
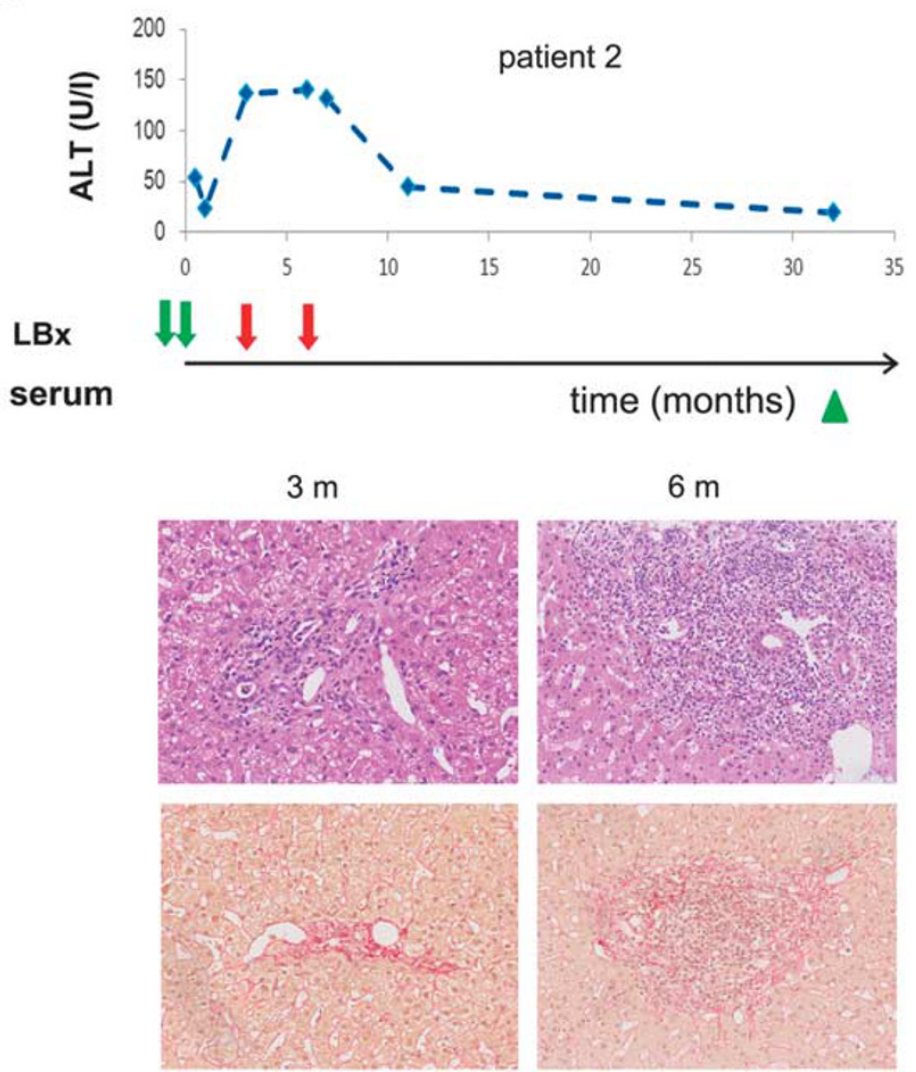

b

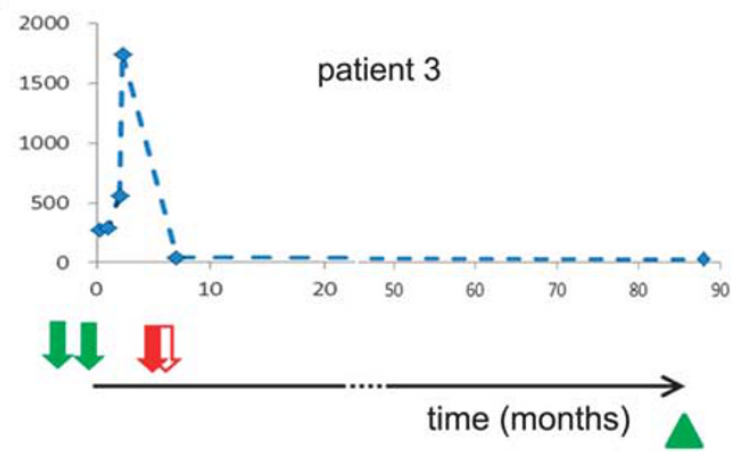

$2 \mathrm{~m}$

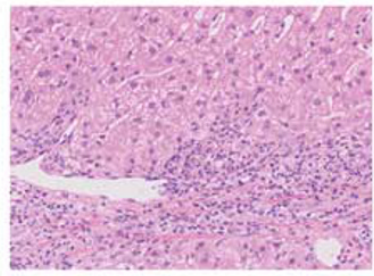

$3 \mathrm{~m}$
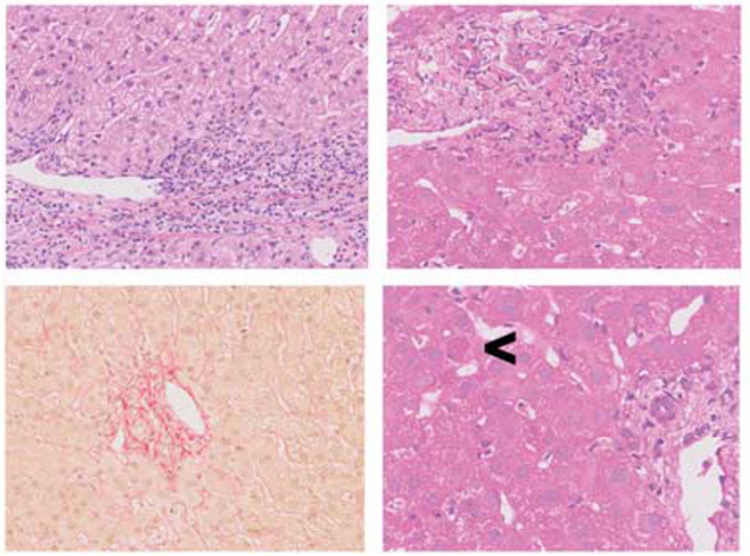

Figure 3 Clinical course and histopathological patterns of hepatitis E virus (HEV) infection after liver transplantation for patients 2 and 3. (a) Patient 2: Elevated alanine aminotransferase (ALT) and also aspartate aminotransferase (AST) levels prompted liver biopsies at 3 and 6 months post liver transplantation. HEV infection was first detected the liver biopsy 3 months post liver transplantation. Transaminase levels normalized, follow-up serological testing was negative. Liver histology at 3 and 6 months post LT revealed mainly portal hepatitis with spotty hepatocyte necrosis, but no significant fibrosis. (b) Patient 3: Elevated ALT and AST levels prompted liver biopsies at 2 and 2.5 months post liver transplantation. HEV infection was first detected in the liver biopsy 2 months post liver transplantation. Transaminase levels normalized, follow-up serological testing was negative. Liver histology at 2 months after liver transplantation revealed signs of acute cellular rejection. After treatment, features of acute cellular rejection regressed, but spotty hepatocyte necroses persisted. Arrow: liver biopsy, arrow head: serum testing; red: positive for HEV, green: negative for HEV.
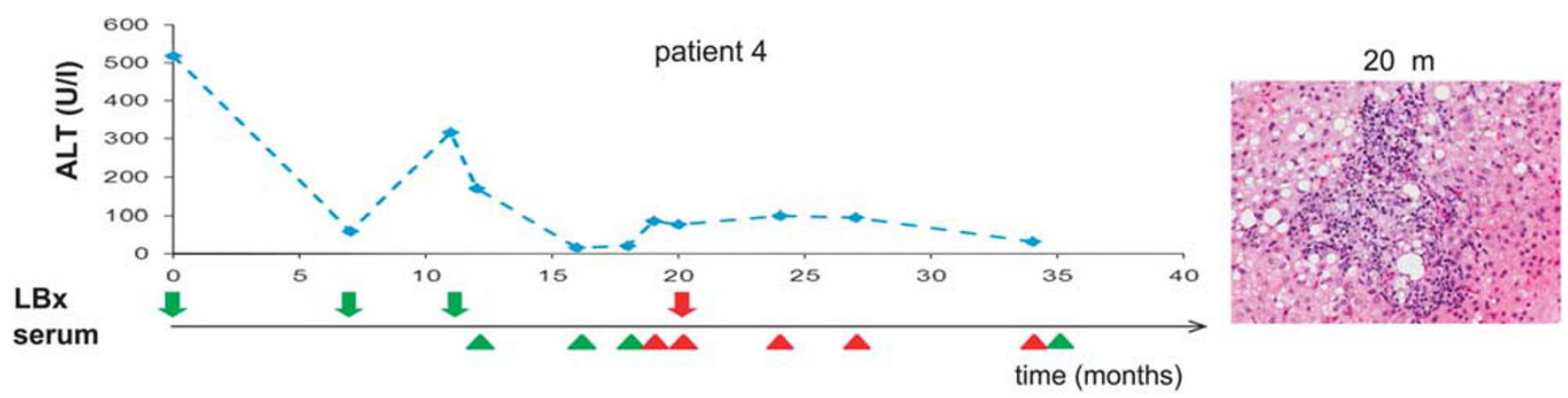

Figure 4 Clinical course and histopathological patterns of hepatitis E virus (HEV) infection after liver transplantation for patient 4. Elevated alanine aminotransferase (ALT) and aspartate aminotransferase (AST) levels prompted liver biopsy 20 months post liver transplantation in which HEV was first detected. Transaminase levels normalized, follow-up serologic testing was repeatedly positive, but the last testing (35 months post liver transplantation) was negative. Liver histology at 20 months after liver transplantation revealed inflammation in some portal tracts with focal interface activity. Arrow: liver biopsy, arrow head: serum testing; red: positive for HEV, green: negative for HEV.

\section{Discussion}

Recently, locally acquired (autochthonous) HEV infections have been increasingly observed in developed countries. These become relevant in particular in immunosuppressed patients especially after solid organ transplantation when chronic courses may occur and in the case of liver transplantation the transplanted organ may be damaged. We developed a sensitive method to test 
for HEV infection in liver biopsies and used it to retrospectively analyze the incidence of HEV infection in liver transplantation patients in two large European centers. By this, previously undetected HEV infections were found in two patients. The presented study is the first investigation in which systematic molecular testing for HEV infection was performed in liver tissues of a substantial number of patients complemented by a detailed histological analysis.

The (semi-nested)-RT-PCR protocol for the detection of HEV was designed to detect all known HEV genotypes and to be applicable also on formalinfixed, paraffin-embedded specimens. In contrast to most liquid and fresh samples, formalin-fixed, paraffin-embedded tissue is routinely archived, and sometimes the only material available for testing. Using this protocol, we detected HEV RNA in liver biopsies of 4 out of 282 patients during a medium observation period of 16 months after LT corresponding to $1 \%$ of liver biopsies. Sequencing confirmed detection of HEV and excluded false positive test results, so that we assume the protocol to be robust and sufficiently sensitive and thus suitable for routine diagnostic application on fresh and formalin-fixed, paraffin-embedded samples.

So far no data are available that allow comparing the sensitivity and specificity of HEV testing in blood samples vs testing in liver biopsies. Due to sample availability and local regulations, we were not able to perform additional retrospective testing of blood samples from all patients. However, systematic retrospective analyses of serum samples of a subcohort of 159 patients from the German center allowed for comparing serum vs tissue testing. Testing for anti-HEV antibodies revealed seropositivity in $18 \%$ of those patients who underwent liver transplantation. Importantly, 3\% (5/159 patients) had acquired HEV infection shortly before or after liver transplantation. Four patients had self-limiting infection and had cleared HEV before a liver biopsy was taken. In one patient persistent infection developed, which was also detected in formalin-fixed, paraffin-embedded liver tissue. Out of the 123 patients from the Swiss center from whom only liver biopsies were available, three $(2 \%)$ were diagnosed with HEV infection after liver transplantation. Taken together the results of serological and liver tissue testing, we found an incidence of $3 \%$ of de novo HEV infection during the 1- to 8-year follow-up in our cohort.

Inherent with their retrospective nature, all studies including our have limitations in assessing the exact prevalence rate due to variable inclusion criteria. In the study by Haagsma et $a l,{ }^{15}$ the initial screen was restricted to the most recent serum sample most likely leading to underestimation of prevalence since infections lacking seroconversion may have been missed. Our study was limited since not all liver transplantation patients were tested, but only those who had a liver biopsy 4 weeks or later post liver transplantation. This approach has a twofold bias on the detection rate with opposite effects: On the one hand, it selects for patients who showed liver abnormalities; on the other hand, it misses cases of clinically silent infections and those in which a biopsy was not performed. This was exemplified by the three patients, in whom retrospective serum testing revealed self-limited HEV infection after liver transplantation, which did not initiate a liver biopsy at the time of infection. Direct site by site comparison of serological and liver tissue testing for HEV RNA in our cohort was limited since sera were always available from the time of liver biopsy, but not vice versa. Independent of the variable inclusion criteria and diverse assays applied in the different studies, a rate of $1-2 \%$ de novo $\mathrm{HEV}$ infection after liver transplantation was consistently found. With nearly $3 \%$ we found an even higher rate of de novo HEV infections in our cohort, probably due to the combined testing of sera and liver biopsy we performed here. This confirms that HEV infections after liver transplantation are relevant and underlines that HEV infection should be among the differential diagnoses when liver abnormalities of unexplained origin arise after liver transplantation.

Sequencing of the PCR amplicons revealed HEV genotype 3 in all patients with de novo $\mathrm{HEV}$ infection. This is in line with findings of other studies in which HEV genotype 3 consistently was identified, ${ }^{14,15,23}$ indicating community-acquired zoonotic HEV infection. Nucleotide sequence comparison revealed inter-, but no intra-individual sequence variability. From a technical perspective, this confirmed the specificity of PCR amplification and excluded a laboratory contamination as the source of positive testing. From a clinical perspective, this finding makes it less likely that patients had the same origin of infection, which was conceivable taking into account that they were treated in the same centers partially at overlapping time periods. Furthermore, although the informative value of the sequence analysis is limited to a 228-bp stretch of the HEV ORF 2/3 gene region, the fact that inter-, but no intra-individual sequences variability was found suggested a limited selection pressure in the infected individual. ${ }^{24}$

How do clinical parameters of the four patients identified in this study with HEV infection post liver transplantation relate to what is known so far? The rate of chronic courses in HEV-infected patients after solid organ transplantation in a recently published single-center retrospective study was determined as $66 \% .^{23}$ One (patient 1) of the four patients in our study clearly developed chronic hepatitis, and two (patient 2 and patient 4) had viral persistence for more than 6 months. Patient 3 was the one with highest transaminase levels (asparate aminotransferase (AST): $515 \mathrm{U} / \mathrm{l}$; alanine aminotransferase (ALT): $1743 \mathrm{U} / \mathrm{l}$ ) and fastest viral clearance which is notable since low (peak) transaminase levels have been described as a factor 
associated with the development of chronicity. ${ }^{23}$ All four patients had at least temporarily received tacrolimus, which has been shown to be an independent risk factor for a chronic course. ${ }^{23}$ Patient 1 , patient 2, and patient 3 acquired HEV at a remarkably early time post liver transplantation (first detectable 8,3 , and 2 months post liver transplantation, respectively) which is considered as a risk factor for the development of chronicity. ${ }^{14,23}$ Some reports indicate that therapy with ribavirin $^{22,25}$ or pegylated interferon ${ }^{21}$ may be beneficial to achieve HEV clearance in immunosuppressed patients. Patient 1 also might have benefited from treatment.

Histological features comprising a spectrum of changes characteristic for viral hepatitis were variably present and changed during the course of infection. Initial HEV-positive biopsies most likely reflect the acute phase of infection. Remarkably, in contrast to what is described for acute autochthonous hepatitis $\mathrm{E}$ infection in immunocompetent individuals, ${ }^{26,27}$ cholestasis, bilirubinostasis, or lobular disarray was not among the histological features of (acute) HEV infection seen in the four immunosuppressed patients of this study. Besides histological changes characteristic for viral hepatitis, we also observed untypical findings: The first HEV-positive biopsy of pt1 revealed only few hepatocellular apoptoses, but no significant inflammation. Additional features of mild acute cellular rejection were found in patient 2 and patient 3 . Thus, histological changes in HEV hepatitis after liver transplantation in the early phase of infection were modified or superimposed by immunosuppressive therapy and/or other medication. In the later course of infection, typical histological features of chronic viral hepatitis such as predominantly portal inflammation and progressive fibrosis were found. ${ }^{14,16,28}$ Our observations illustrate that not only clinical presentation, but also liver histology in cases of HEV under immunosuppression is far from characteristic or even specific. Therefore, histological findings consistent with viral hepatitis should prompt diagnostic workup for HEV.

In conclusion, this retrospective two-center study is the first systematic investigation on the prevalence of HEV infection after liver transplantation determined by testing of liver tissues for HEV RNA. We successfully established molecular testing for HEV RNA applicable on liver biopsies under routine diagnostic conditions, and found a rate of de novo HEV infection of $3 \%$ after liver transplantation. Clinical findings of this study added to the knowledge on HEV infection under immunosuppression, and thorough histological analyses revealed that morphology especially during early phases of infection can be uncharacteristic and is distinct from acute HEV infection in immunocompetent patients. Our data argue for systematic HEV testing in liver transplantation patients with elevated transaminase levels in serum, which is possible also in liver biopsies.

\section{Acknowledgments}

We would like to thank the staff members of the BioMaterial Bank Heidelberg (Nationale Centrum für Tumorerkrankungen (NCT) and Deutsches Zentrum für Infektionsforschung (DZIF) tissue bank), University of Heidelberg, for providing access to archival material.

\section{Disclosure/conflict of interest}

The authors declare no conflict of interest.

\section{References}

1 Aggarwal R, Jameel S. Hepatitis E. Hepatology 2011;54:2218-2226.

2 Aggarwal R. Hepatitis E: Historical, contemporary and future perspectives. J Gastroenterol Hepatol 2011; 26(Suppl 1):72-82.

3 Wedemeyer H, Pischke S, Manns MP. Pathogenesis and treatment of hepatitis e virus infection. Gastroenterology 2012;142:1388-1397; e1.

4 Kamar N, Bendall R, Legrand-Abravanel F, et al. Hepatitis E. Lancet 2012;379:2477-2488.

5 Hoofnagle JH, Nelson KE, Purcell RH. Hepatitis E. N Engl J Med 2012;367:1237-1244.

6 Bihl F, Negro F. Hepatitis E virus: a zoonosis adapting to humans. J Antimicrob Chemother 2010;65:817-821.

7 Dalton HR, Bendall R, Ijaz S, Banks M. Hepatitis E: an emerging infection in developed countries. Lancet Infect Dis 2008;8:698-709.

8 Dalton HR, Stableforth W, Hazeldine S, et al. Autochthonous hepatitis $\mathrm{E}$ in Southwest England: a comparison with hepatitis A. Eur J Clin Microbiol Infect Dis 2008;27:579-585.

9 Dalton HR, Fellows HJ, Stableforth W, et al. The role of hepatitis E virus testing in drug-induced liver injury. Aliment Pharmacol Ther 2007;26:1429-1435.

10 Davern TJ, Chalasani N, Fontana RJ, et al. Acute hepatitis $\mathrm{E}$ infection accounts for some cases of suspected drug-induced liver injury. Gastroenterology 2011;141:1665-1672; e1-9.

11 Bhatia V, Singhal A, Panda SK, Acharya SK. A 20-year single-center experience with acute liver failure during pregnancy: is the prognosis really worse? Hepatology 2008;48:1577-1585.

12 Kumar Acharya S, Kumar Sharma P, Singh R, et al. Hepatitis E virus (HEV) infection in patients with cirrhosis is associated with rapid decompensation and death. J Hepatol 2007;46:387-394.

13 Peron JM, Bureau C, Poirson H, et al. Fulminant liver failure from acute autochthonous hepatitis $\mathrm{E}$ in France: description of seven patients with acute hepatitis E and encephalopathy. J Viral Hepat 2007;14:298-303.

14 Kamar N, Selves J, Mansuy JM, et al. Hepatitis E virus and chronic hepatitis in organ-transplant recipients. N Engl J Med 2008;358:811-817.

15 Haagsma EB, Niesters HG, van den Berg AP, et al. Prevalence of hepatitis $\mathrm{E}$ virus infection in liver 
transplant recipients. Liver Transpl 2009;15: 1225-1228.

16 Haagsma EB, van den Berg AP, Porte RJ, et al. Chronic hepatitis $\mathrm{E}$ virus infection in liver transplant recipients. Liver Transpl 2008;14:547-553.

17 Syn WK, Nightingale P, Gunson B, Hubscher SG, Neuberger JM. Natural history of unexplained chronic hepatitis after liver transplantation. Liver Transpl 2007;13:984-989.

18 Bendall R, Ellis V, Ijaz S, Ali R, Dalton H. A comparison of two commercially available anti-HEV IgG kits and a re-evaluation of anti-HEV IgG seroprevalence data in developed countries. J Med Virol 2010;82:799-805.

19 Baylis SA, Hanschmann KM, Blumel J, Nubling CM. Standardization of hepatitis E virus (HEV) nucleic acid amplification technique-based assays: an initial study to evaluate a panel of HEV strains and investigate laboratory performance. J Clin Microbiol 2011;49: 1234-1239.

20 Banff. Banff schema for grading liver allograft rejection: an international consensus document. Hepatology 1997;25:658-663.

21 Kamar N, Abravanel F, Garrouste C, et al. Three-month pegylated interferon-alpha-2a therapy for chronic hepatitis E virus infection in a haemodialysis patient. Nephrol Dial Transplant 2010;25:2792-2795.
22 Kamar N, Izopet J, Tripon S, et al. Ribavirin for chronic hepatitis $\mathrm{E}$ virus infection in transplant recipients. $\mathrm{N}$ Engl J Med 2014;370:1111-1120.

23 Kamar N, Garrouste C, Haagsma EB, et al. Factors associated with chronic hepatitis in patients with hepatitis $\mathrm{E}$ virus infection who have received solid organ transplants. Gastroenterology 2011;140: 1481-1489.

24 Hoffmann D, Hutzenthaler M, Seebach J, et al. Norovirus GII.4 and GII.7 capsid sequences undergo positive selection in chronically infected patients. Infect Genet Evol 2012;12:461-466.

25 Kamar N, Rostaing L, Abravanel F, et al. Ribavirin therapy inhibits viral replication on patients with chronic hepatitis e virus infection. Gastroenterology 2010;139:1612-1618.

26 Malcolm P, Dalton H, Hussaini HS, Mathew J. The histology of acute autochthonous hepatitis E virus infection. Histopathology 2007;51:190-194.

27 Peron JM, Danjoux M, Kamar N, et al. Liver histology in patients with sporadic acute hepatitis E: a study of 11 patients from South-West France. Virchows Arch 2007;450:405-410.

28 Kamar N, Abravanel F, Selves J, et al. Influence of immunosuppressive therapy on the natural history of genotype 3 hepatitis-E virus infection after organ transplantation. Transplantation 2010;89:353-360. 